\title{
ON THE ORDER OF MAXIMUM ERROR OF THE FINITE DIFFERENCE SOLUTIONS OF LAPLACE'S EQUATION ON RECTANGLES
}

\author{
A. A. DOSIYEV ${ }^{\bowtie 1}$ and S. CIVAL BURANAY ${ }^{2}$
}

(Received 4 May, 2007; revised 28 April, 2008)

\begin{abstract}
The finite difference solution of the Dirichlet problem on rectangles when a boundary function is given from $C^{1,1}$ is analyzed. It is shown that the maximum error for a ninepoint approximation is of the order of $O\left(h^{2}(|\ln h|+1)\right)$ as a five-point approximation. This order can be improved up to $O\left(h^{2}\right)$ when the nine-point approximation in the grids which are a distance $h$ from the boundary is replaced by a five-point approximation ("five and nine"-point scheme). It is also proved that the class of boundary functions $C^{1,1}$ used to obtain the error estimations essentially cannot be enlarged. We provide numerical experiments to support the analysis made. These results point at the importance of taking the smoothness of the boundary functions into account when choosing the numerical algorithms in applied problems.
\end{abstract}

2000 Mathematics subject classification: 65N06, 65N15.

Keywords and phrases: finite difference method, nonsmooth solutions, uniform error.

\section{Introduction}

In many versions of the domain decomposition, composite grids and combined methods in solving Laplace's boundary value problems, the obtained system of equations is separated into a fixed number of subsystems, each of which is adequate for the difference equations on a rectangle (see [2, 3, 5, 6, 8, 10, 14]). Therefore, a detailed error analysis becomes important for the classical finite difference or finite element methods for the problems in rectangular domains. It is also known that, to enlarge a class of problems to apply the above-mentioned methods, the maximum possible order of accuracy should be obtained by minimum requirements on the functions given in the boundary conditions (see, for example, $[1,4,12,14]$ ).

\footnotetext{
${ }^{1}$ Department of Mathematics, Eastern Mediterranean University, Gazimagusa, Cyprus, Mersin 10, Turkey; e-mail: adiguzel.dosiyev@emu.edu.tr.

${ }^{2}$ Department of Mathematics, Eastern Mediterranean University, Gazimagusa, Cyprus, Mersin 10, Turkey; e-mail: suzan.buranay@emu.edu.tr.

(C) Australian Mathematical Society 2008, Serial-fee code 1446-1811/08 \$16.00
} 
In [12] the five-point scheme on square grids used to solve the Dirichlet problem for Laplace's equation on rectangles was analyzed. It was proved that, when the boundary function is from $C^{1,1}$, that is, it has the first derivative that satisfies a Lipschitz condition, the error in maximum norm is of the order of $O\left(h^{2}(|\ln h|+1)\right),(h$ is the mesh step), and the requirements on the boundary functions cannot be lowered.

In this paper, we analyze the classical nine-point scheme on square grids for the same class of boundary functions as in [12], that is, from $C^{1,1}$. It is shown (Section 4) that the nine-point scheme cannot improve the order of accuracy in maximum norm for the same class of boundary functions $C^{1,1}$ as in the five-point scheme. Moreover, it is proved that $O\left(h^{2}(|\ln h|+1)\right)$ order accuracy cannot be obtained for the class of boundary functions $C^{1, \lambda}, 0<\lambda<1$, that is, the requirements on the boundary functions cannot be lowered in Hölder classes $C^{k, \lambda}$. In Section 5 of this paper, it is proved that, if we replace the nine-point approximation with a simple five-point approximation ("five and nine"-point scheme) just on the grids which are a distance $h$ from the boundary of the rectangle, the order of accuracy in the maximum norm becomes $O\left(h^{2}\right)$, that is, the factor $|\ln h|$ is removed.

In Section 2 of this paper, the necessary differential properties for the solution of Laplace's equation are given. In Section 3, we formulate the existing error estimations in uniform metric.

\section{The Drichlet problem on rectangular domains}

Let

$$
\Pi=\{(x, y) \mid 0<x<a, 0<y<b\}
$$

be a rectangle, $a / b$ be rational, $\gamma_{j}, j=1,2,3,4$, be the sides, including the ends, enumerated counterclockwise starting from the left side $\left(\gamma_{0} \equiv \gamma_{4}, \gamma_{5} \equiv \gamma_{1}\right)$, and let $\gamma=\bigcup_{j=1}^{4} \gamma_{j}$, be the boundary of $\Pi$. Denote by $s$ the arc length, measured along the $\gamma$, and denote by $s_{j}$ the beginning of $\gamma_{j}$.

We consider the boundary value problem

$$
\Delta u=0 \quad \text { on } \Pi, \quad u=\varphi_{j}(s) \quad \text { on } \gamma_{j}, j=1,2,3,4,
$$

where $\Delta \equiv \partial^{2} / \partial x^{2}+\partial^{2} / \partial y^{2}, \varphi_{j}$ is a given continuous function of the arc length $s$ taken along $\gamma$.

We give the following definitions and theorems which will be used in the following sections for the analysis of the finite difference solutions.

Definition 2.1. We say that $f \in C^{k, \lambda}(D)$, if $f$ has $k$ th derivatives on $D$ satisfying a Hölder condition with exponent $\lambda$.

THEOREM 2.2. The solution of the problem (2.1) $u \in C^{k, \lambda}(\bar{\Pi}), k \geq 0,0<\lambda<1$, if and only if

$$
\varphi_{j}(s) \in C^{k, \lambda}\left(\gamma_{j}\right)
$$


and for $s=s_{j}$ the conjugation conditions

$$
\varphi_{j}^{(2 q)}=(-1)^{q} \varphi_{j-1}^{(2 q)},
$$

are satisfied, where $q=0,1, \ldots, Q, Q=[k / 2], j=1,2,3,4$.

ProOF. See [11, Theorems 3.1 and 3.2].

DeFinition 2.3. We say that the solution $u$ of the problem (2.1) belongs to $\widetilde{C}^{k, \lambda}(\bar{\Pi})$, $k \geq 2,0<\lambda<1$, if (2.2) and (2.3) are satisfied when $Q=[k / 2]-1$.

Definition 2.4. We say that the boundary function $\varphi_{j} \in C^{1,1}\left(\gamma_{j}\right)$ if $\varphi_{j}$ has first derivative, which satisfies a Lipschitz condition on $\gamma_{j}, j=1,2,3,4$.

THEOREM 2.5. Let $\varphi_{j} \in C^{1,1}\left(\gamma_{j}\right)$ and let $\varphi_{j-1}\left(s_{j}\right)=\varphi_{j}\left(s_{j}\right), j=1,2,3,4$, then the following statements for the derivatives of the solution of the problem (2.1) on $\Pi$ are true.

(a) The second-order pure derivatives are bounded, that is,

$$
\sup _{\Pi}\left\{\left|\frac{\partial^{2} u}{\partial x^{2}}\right|,\left|\frac{\partial^{2} u}{\partial y^{2}}\right|\right\} \leq \Phi,
$$

where $\Phi$ is a constant which is defined by the Lipschitz coefficients of the first derivative of the boundary functions $\varphi_{j}, j=1,2,3,4$.

(b) The inequalities

$$
\begin{gathered}
\left|\frac{\partial^{2} u(x, y)}{\partial x \partial y}\right| \leq \widetilde{c}_{2}(|\ln t|+1), \\
\left|\frac{\partial^{p} u(x, y)}{\partial x^{q} \partial y^{p-q}}\right| \leq \widetilde{c}_{p} t^{2-p}, \quad p>2,0 \leq q \leq p,
\end{gathered}
$$

are satisfied for the mixed derivatives, where $t$ is the distance from $(x, y) \in \Pi$ to $\gamma$ (boundary of $\Pi$ ), and $\widetilde{c}_{\nu}, v \geq 2$, are constants that are independent of $t$.

PROOF. The proof follows from [12, Theorems 4.1 and 6.1], and from [13, Estimations (2.8) and (2.9)].

\section{Finite difference solutions}

Let $h>0$, and $a / h \geq 4, b / h \geq 4$ be integers. We assign $\Pi^{h}$, a square net on $\Pi$, with step $h$, obtained with the lines $x, y=0, h, 2 h, \ldots$ Let $\gamma_{j}^{h}$ be a set of nodes on the interior of $\gamma_{j}$, and let

$$
\begin{gathered}
\gamma^{h}=\bigcup_{j=1}^{4} \gamma_{j}^{h}, \quad \dot{\gamma}_{j}=\gamma_{j-1} \cap \gamma_{j}, \quad \bar{\gamma}^{h}=\bigcup_{j=1}^{4}\left(\gamma_{j}^{h} \cup \dot{\gamma}_{j}\right), \\
\bar{\Pi}^{h}=\Pi^{h} \cup \bar{\gamma}^{h}, \quad \widetilde{\Pi}^{h}=\Pi^{h} \cup \gamma^{h} .
\end{gathered}
$$


We denote by $\Pi^{1 h}$ the set of such nodes of $\Pi^{h}$ that are a distance $h$ from $\gamma$ and $\Pi^{2 h}=\Pi^{h} \backslash \Pi^{1 h}$.

Let the operators $A$ and $B$ be defined as follows

$$
\begin{aligned}
A u(x, y)= & (u(x+h, y)+u(x-h, y)+u(x, y+h)+u(x, y-h)) / 4, \\
B u(x, y)= & (u(x+h, y)+u(x-h, y)+u(x, y+h)+u(x, y-h)) / 5 \\
& +(u(x+h, y+h)+u(x+h, y-h) \\
& +u(x-h, y+h)+u(x-h, y-h)) / 20 .
\end{aligned}
$$

We consider the following two classical finite difference approximations of the problem (2.1):

(i) five-point approximation

$$
u_{h}^{A}=A u_{h}^{A} \quad \text { on } \Pi^{h}, \quad u_{h}^{A}=\varphi_{j} \quad \text { on } \gamma_{j}^{h}, j=1,2,3,4
$$

(ii) nine-point approximation

$$
u_{h}^{B}=B u_{h}^{B} \quad \text { on } \Pi^{h}, \quad u_{h}^{B}=\varphi_{j} \quad \text { on } \gamma_{j}^{h} \cup \dot{\gamma}_{j}, j=1,2,3,4 .
$$

In the following, for simplicity we denote the constants which are independent of $h$ by $c, c_{0}, c_{1}, \ldots$.

Let $u_{h}^{A}$ and $u_{h}^{B}$ be the solutions of the finite difference problems (3.3) and (3.4), respectively, and let $u$ be the solution of problem (2.1).

The following theorems hold.

THEOREM 3.1 (Volkov [14]). If the solution to the problem (2.1) $u \in \widetilde{C}^{2, \lambda}(\bar{\Pi})$, $0<\lambda<1$, then

$$
\max _{\widetilde{\Pi}^{h}}\left|u_{h}^{A}-u\right| \leq c h^{2}
$$

THEOREM 3.2 (Dosiyev [4]). If the solution of the problem (2.1) $u \in \widetilde{C}^{6, \lambda}(\bar{\Pi})$, $0<\lambda<1$, then

$$
\max _{\bar{\Pi}^{h}}\left|u_{h}^{B}-u\right| \leq c h^{6}
$$

Theorem 3.3 (Volkov [12]). If $\varphi_{j} \in C^{1,1}\left(\gamma_{j}\right)$ and $\varphi_{j-1}\left(s_{j}\right)=\varphi_{j}\left(s_{j}\right), j=1,2,3,4$, then

$$
\max _{\widetilde{\Pi}^{h}}\left|u_{h}^{A}-u\right| \leq c h^{2}(|\ln h|+1) .
$$

\section{Nine-point solution on rectangles}

We show that Theorem 3.3 holds for the nine-point solution as well.

THEOREM 4.1. If $\varphi_{j} \in C^{1,1}\left(\gamma_{j}\right)$ and $\varphi_{j-1}\left(s_{j}\right)=\varphi_{j}\left(s_{j}\right), j=1,2,3,4$, then

$$
\max _{\bar{\Pi}^{h}}\left|u_{h}^{B}-u\right| \leq c_{0} h^{2}(|\ln h|+1)+c h^{2} .
$$


PROOF. Let

$$
\varepsilon_{h}^{B}=u_{h}^{B}-u \quad \text { on } \bar{\Pi}^{h}=\Pi^{1 h} \cup \Pi^{2 h} \cup \bar{\gamma}^{h} .
$$

It is obvious that

$$
\varepsilon_{h}^{B}=B \varepsilon_{h}^{B}+(B u-u) \quad \text { on } \Pi^{h}, \quad \varepsilon_{h}^{B}=0 \quad \text { on } \bar{\gamma}^{h} .
$$

Let $(x, y) \in \Pi^{1 h}$ then $(x+s H, y+s K) \in \bar{\Pi}$, for $0 \leq s \leq 1, H, K=-h, 0, h$, $H^{2}+K^{2}>0$. We use the next form of Taylor's formula to estimate $B u-u$ on $\Pi^{1 h}$

$$
\begin{aligned}
u(x+ & H, y+K) \\
= & u(x, y)+H u_{x}(x, y)+K u_{y}(x, y)+\int_{0}^{1}(1-q)\left[H^{2} u_{x x}(x+q H, y+q K)\right. \\
& \left.+2 H K u_{x y}(x+q H, y+q K)+K^{2} u_{y y}(x+q H, y+q K)\right] d q .
\end{aligned}
$$

On the basis of (2.4) and (2.5) the absolute value of the integral in (4.4) is estimated by

$$
4 \widetilde{c}_{2} h^{2} \int_{0}^{1}(1-q)|\ln h(1-q)| d q \leq c_{1} h^{2}(|\ln h|+1) .
$$

By virtue of (3.2), (4.4) and (4.5), we obtain

$$
|B u-u| \leq c_{2} h^{2}(|\ln h|+1) \quad \text { on } \Pi^{1 h} .
$$

We represent the solution of (4.3) as

$$
\varepsilon_{h}^{B}=\varepsilon_{h}^{1}+\varepsilon_{h}^{2},
$$

where

$$
\begin{array}{ccccc}
\varepsilon_{h}^{1}=B \varepsilon_{h}^{1}+B u-u & \text { on } \Pi^{1 h}, \quad \varepsilon_{h}^{1}=B \varepsilon_{h}^{1} & \text { on } \Pi^{2 h}, & \varepsilon_{h}^{1}=0 & \text { on } \bar{\gamma}^{h} ; \\
\varepsilon_{h}^{2}=B \varepsilon_{h}^{2} & \text { on } \Pi^{1 h}, \quad \varepsilon_{h}^{2}=B \varepsilon_{h}^{2}+B u-u & \text { on } \Pi^{2 h}, & \varepsilon_{h}^{2}=0 & \text { on } \bar{\gamma}^{h} .
\end{array}
$$

On the basis of (4.6), (4.8), and the principle of the maximum (see [9, Ch. 4]) for the solution $\varepsilon_{h}^{1}$ of the problem (4.8),

$$
\max _{\bar{\Pi}^{h}}\left|\varepsilon_{h}^{1}\right| \leq \frac{10}{3} c_{2} h^{2}(|\ln h|+1) .
$$

We show that

$$
\max _{\bar{\Pi}^{h}}\left|\varepsilon_{h}^{2}\right| \leq \frac{10}{3} \max _{\bar{\Pi}^{h}}\left|\varepsilon_{h}^{3}\right|
$$

where

$$
\varepsilon_{h}^{3}=0 \quad \text { on } \Pi^{1 h} \cup \bar{\gamma}^{h}, \quad \varepsilon_{h}^{3}=B \varepsilon_{h}^{3}+B u-u \quad \text { on } \Pi^{2 h} .
$$


Let $\tau_{h}$ be a solution of the problem

$$
\tau_{h}=\varepsilon_{h}^{2} \quad \text { on } \Pi^{1 h}, \quad \tau_{h}=B \tau_{h} \quad \text { on } \Pi^{2 h}, \quad \tau_{h}=0 \quad \text { on } \bar{\gamma}^{h} .
$$

It is obvious that

$$
\varepsilon_{h}^{2}=\varepsilon_{h}^{3}+\tau_{h}
$$

By virtue of (4.9) and (4.13),

$$
\max _{\bar{\Pi}^{h}}\left|\tau_{h}\right| \leq \frac{7}{10} \max _{\bar{\Pi}^{h}}\left|\varepsilon_{h}^{2}\right| .
$$

The inequality (4.11) follows from (4.14) and (4.15).

Now, we estimate the solution of the problem (4.12). Let $\Pi_{k}^{2 h}, 2 \leq k \leq\left[a^{*} / 2 h\right]$, $a^{*}=\min \{a, b\}$, be the subsets of $\Pi^{2 h}$, such that, the distance from each point of $\Pi^{2 h}$ to $\gamma$ is equal to $k h$, and let $\Pi_{1}^{2 h} \equiv \Pi^{1 h}, \Pi_{0}^{2 h} \equiv \bar{\gamma}^{h}$. According to the Taylor formula with the remainder term in Lagrange form expressing eighth derivatives and by using the estimation (2.6) for $p=8$,

$$
|B u-u| \leq \frac{c_{3} h^{8}}{(k h)^{6}} \quad \text { on } \Pi_{k}^{2 h}, \quad 2 \leq k \leq\left[a^{*} / 2 h\right] .
$$

We define the functions $\Psi_{k}(P), 2 \leq k \leq\left[a^{*} / 2 h\right], P \in \bar{\Pi}^{h}$ as follows

$$
\Psi_{k}(P)= \begin{cases}\frac{10 m}{3} & \text { if } P \in \Pi_{m}^{2 h}, 0 \leq m \leq k \\ \frac{10 k}{3} & \text { if } P \in \Pi_{m}^{2 h}, m>k\end{cases}
$$

It is easy to show that the functions $\Psi_{k}(P), 2 \leq k \leq\left[a^{*} / 2 h\right]$, are solutions of the finite difference problem

$$
\begin{gathered}
\Psi_{k}=B \Psi_{k}+q_{k} \quad \text { on } \Pi_{k}^{2 h}, \quad \Psi_{k}=B \Psi_{k} \quad \text { on } \Pi^{2 h} \backslash \Pi_{k}^{2 h}, \\
\Psi_{k}=\frac{10}{3} \quad \text { on } \Pi_{1}^{2 h}, \quad \Psi_{k}=0 \quad \text { on } \Pi_{0}^{2 h},
\end{gathered}
$$

where $q_{k},\left(1 \leq q_{k} \leq(10 / 3)\right)$ are real numbers.

Let $\varepsilon_{h, k}^{3}$ be the solution to the problem

$$
\begin{gathered}
\varepsilon_{h, k}^{3}=B \varepsilon_{h, k}^{3}+r_{h, k} \quad \text { on } \Pi_{k}^{2 h}, \quad \varepsilon_{h, k}^{3}=B \varepsilon_{h, k}^{3} \quad \text { on } \Pi^{2 h} \backslash \Pi_{k}^{2 h}, \\
\varepsilon_{h, k}^{3}=0 \quad \text { on } \Pi^{1 h} \cup \bar{\gamma}^{h},
\end{gathered}
$$

where $2 \leq k \leq\left[a^{*} / 2 h\right]$,

$$
r_{h, k}=c_{3} \frac{h^{2}}{k^{6}}
$$


On the basis of (4.18)-(4.20) and the comparison theorem (see [9, Ch. 4]),

$$
\varepsilon_{h, k}^{3} \leq c_{3} \frac{h^{2}}{k^{6}} \Psi_{k}, \quad 2 \leq k \leq\left[a^{*} / 2 h\right] .
$$

Furthermore, by virtue of (4.12), (4.17), (4.19) and (4.21), we obtain

$$
\max _{\bar{\Pi}^{h}}\left|\varepsilon_{h}^{3}\right| \leq \sum_{k=2}^{\left[a^{*} / 2 h\right]} \varepsilon_{h, k}^{3} \leq \sum_{k=2}^{\left[a^{*} / 2 h\right]} c_{3} \frac{h^{2}}{k^{6}} \Psi_{k} \leq \frac{10}{3} c_{3} h^{2} \sum_{k=2}^{\left[a^{*} / 2 h\right]} \frac{1}{k^{5}} \leq c_{4} h^{2} .
$$

From (4.7), (4.10), (4.11) and (4.22),

$$
\max _{\bar{\Pi}^{h}}\left|\varepsilon_{h}^{B}\right| \leq \max _{\bar{\Pi}^{h}}\left|\varepsilon_{h}^{1}\right|+\max _{\bar{\Pi}^{h}}\left|\varepsilon_{h}^{2}\right| \leq c_{5} h^{2}(|\ln h|+1)+c_{6} h^{2} .
$$

The estimation (4.1) is thus proved.

THEOREM 4.2. The class of boundary functions in Theorem 4.1 cannot be enlarged in Hölder classes $C^{k, \lambda}$.

ProOf. Let $\Pi=\{(x, y) \mid 0<x<a,-b / 2<y<b / 2\}$, and let $\gamma$ be its boundary. We consider the harmonic function

$$
u=r^{1+\lambda} \cos (1+\lambda) \theta \text { on } \Pi,
$$

where $1 / 2<\lambda<1, r=|z|, \theta=\arg z, z=x+i y$. It is obvious that $u \in C^{1, \lambda}(\gamma)$.

Let $h>0, a / h, b / h$ be integers, and the net $\Pi^{h}$ be constructed by the lines $x=0, h, 2 h, \ldots, y=0, \pm h, \pm 2 h, \ldots$ We show that there exist a real number $h_{0}$ and an integer number $T \geq 2$, such that for all $h \leq h_{0}$ the point $P(T h, 0) \in \Pi^{h}$ and

$$
\mid B u-u \|_{P}>c h^{1+\lambda}, \quad h \leq h_{0} .
$$

On the basis of (3.2) and Taylor's formula with the remainder term $R(u, h)$ expressed through the ninth derivatives, we have (see [7])

$$
\left.(B u-u)\right|_{P}=\left.\frac{4 h^{8}}{8 !} \frac{\partial^{8} u}{\partial x^{4} \partial y^{4}}\right|_{P}+\frac{3 h^{2}}{10} R(u, h) .
$$

For the function (4.24), we obtain

$$
\left.\frac{\partial^{8} u}{\partial x^{4} \partial y^{4}}\right|_{P}>(1-\lambda) \frac{1}{(T h)^{7-\lambda}}
$$

and

$$
\left|\frac{\partial^{9} u}{\partial x^{m} \partial y^{9-m}}\right|<c^{*} \frac{1}{r^{8-\lambda}}, \quad 0 \leq m \leq 9 \quad \text { on } \Pi,
$$

where $c^{*}$ is a constant independent on $r$. 
Since the remainder term $R(u, h)$ contains the values of nine order derivatives with the common factor $h^{7} / 9$ ! at the points on line segments connecting the point $P(T h, 0)$ with the eight neighbor grid points, by taking (4.27) and (4.28) into account, we obtain

$$
|R(u, h)|<\frac{c^{*} h^{7}}{9 !}\left\{\frac{171}{((T-1) h)^{8-\lambda}}+\frac{173}{(T h)^{8-\lambda}}\right\} .
$$

On the basis of (4.26), (4.27) and (4.29) for any $T \geq 2$,

$$
\mid B u-u \|_{P}>\frac{4(1-\lambda) h^{1+\lambda}}{8 ! T^{7-\lambda}}-\frac{3 c^{*} h^{1+\lambda}\left(171 \cdot 2^{8-\lambda}+173\right)}{9 ! 10 T^{8-\lambda}} .
$$

From (4.30) follows that, if we choose $T=2+\left[346 c^{*} /(1-\lambda)\right]$ then the estimation (4.25) is true for all $h \leq h_{0}=a /(T+2)$, and $c=(1-\lambda) /\left(8 ! T^{7-\lambda}\right)$.

Let $u_{h}$ be the solution to the finite difference problem (3.4) when the values of the function $\varphi_{j}$, on $\gamma_{j}^{h} \cup \dot{\gamma}_{j}, j=1,2,3,4$, are replaced with the corresponding values of the function $u$ defined as (4.24). Since

$$
u_{h}-u=B\left(u_{h}-u\right)-(u-B u),
$$

based on (3.2), (3.4), (4.25) from (4.31), we obtain

$$
\max _{\bar{\Pi}^{h}}\left|u_{h}-u\right|>\frac{c}{2} h^{1+\lambda}, \quad 0<h \leq h_{0} .
$$

From (4.32) it follows that if we replace the condition $\varphi_{j} \in C^{1,1}\left(\gamma_{j}\right)$ with $\varphi_{j} \in$ $C^{1, \lambda}\left(\gamma_{j}\right), 1 / 2<\lambda<1$, then the maximum error becomes bounded below by the quantity $h^{1+\lambda}$ on some sequence of values of $h$ tending to zero.

Theorem 4.2 is thus proved.

REMARK 4.3. From the error estimation (4.1) it follows that the term with the $|\ln h|$ factor appears because of the error of approximation of the nine-point scheme on the grid $\Pi^{1 h}$ due to the unboundedness of the second-order mixed derivatives of the exact solution.

\section{5. "Five and nine"-point scheme for the Dirichlet problem with boundary functions from $C^{1,1}$}

We approximate the boundary value problem by the next "five and nine"-point scheme

$$
u_{h}=A u_{h} \quad \text { on } \Pi^{1 h}, \quad u_{h}=B u_{h} \quad \text { on } \Pi^{2 h}, \quad u_{h}=\varphi_{j} \quad \text { on } \gamma_{j}^{h}, j=1,2,3,4 .
$$

THEOREM 5.1. If $\varphi_{j} \in C^{1,1}\left(\gamma_{j}\right)$ and $\varphi_{j-1}\left(s_{j}\right)=\varphi_{j}\left(s_{j}\right), j=1,2,3,4$, then

$$
\max _{\bar{\Pi}^{h}}\left|u_{h}-u\right| \leq c h^{2},
$$


where $u$ is the exact solution of the problem (2.1), $u_{h}$ is the solution of the finite difference problem (5.1), that is, just replacing the nine-point approximation by a fivepoint approximation on $\Pi^{1 h}$ in the approximate problem, the factor $|\ln h|$ from the error estimation (4.1) can be removed.

PRoOF. Let $H$ and $K$ in the formula (4.4) be parameters with the values $-h, 0, h$, and $|H|+|K|=h$. Since $H K=0$, among the second-order derivatives from the expression under the integral sign in the formula (4.4) will be left with one of the pure derivatives only. Then by virtue of the estimation (2.4),

$$
\left|\int_{0}^{1}(1-q)\left(H^{2} u_{x x}(x+q H, y+q K)+K^{2} u_{y y}(x+q H, y+q K)\right) d q\right| \leq \frac{1}{2} \Phi h^{2} .
$$

On the basis of (3.1), (4.4) and (5.3), we obtain

$$
|u-A u| \leq c_{7} h^{2} \quad \text { on } \Pi^{1 h} .
$$

Let

$$
\varepsilon_{h}=u_{h}-u
$$

From (5.1) and (5.5),

$$
\begin{aligned}
& \varepsilon_{h}=A \varepsilon_{h}+A u-u \quad \text { on } \Pi^{1 h}, \\
& \varepsilon_{h}=B \varepsilon_{h}+B u-u \quad \text { on } \Pi^{2 h}, \\
& \varepsilon_{h}=0 \quad \text { on } \gamma_{j}^{h}, \quad j=1,2,3,4 .
\end{aligned}
$$

We represent the solution of system (5.6) as

$$
\varepsilon_{h}=v_{h}^{1}+v_{h}^{2},
$$

where

$$
\begin{array}{ll}
v_{h}^{1}=A v_{h}^{1}+A u-u \quad \text { on } \Pi^{1 h}, \quad v_{h}^{1}=B v_{h}^{1} \quad \text { on } \Pi^{2 h}, \\
v_{h}^{1}=0 \quad \text { on } \gamma_{j}^{h}, j=1,2,3,4 ; \\
v_{h}^{2}=A v_{h}^{2} \quad \text { on } \Pi^{1 h}, \quad v_{h}^{2}=B v_{h}^{2}+B u-u & \text { on } \Pi^{2 h}, \\
v_{h}^{2}=0 \quad \text { on } \gamma_{j}^{h}, j=1,2,3,4 . &
\end{array}
$$

On the basis of (5.4), (5.8) and the principle of maximum,

$$
\max _{\Pi^{h}}\left|v_{h}^{1}\right| \leq \frac{3}{4} \max _{\Pi^{h}}\left|v_{h}^{1}\right|+c_{8} h^{2}
$$

or

$$
\max _{\Pi^{h}}\left|v_{h}^{1}\right| \leq c_{9} h^{2}
$$


By analogy with the inequality (4.11) for the solution of the problem (5.9), we obtain

$$
\max _{\Pi^{h}}\left|v_{h}^{2}\right| \leq 4 \max _{\Pi^{h}}\left|v_{h}^{3}\right|
$$

where

$$
v_{h}^{3}=B v_{h}^{3}+B u-u \quad \text { on } \Pi^{2 h}, \quad v_{h}^{3}=0 \quad \text { on } \Pi^{1 h} \cup \gamma_{h} .
$$

Since $v_{h}^{3} \equiv \varepsilon_{h}^{3}$, where $\varepsilon_{h}^{3}$ is the unique solution of the problem (4.12), from (5.5), (4.22), (5.7), (5.10) and (5.11), we obtain

$$
\max _{\Pi^{h}}\left|\varepsilon_{h}\right| \leq c h^{2}
$$

Theorem 5.1 is thus proved.

REMARK 5.2. On the analogy of the proof of Theorem 4.2, it can be shown that the error estimation (5.2) is obtained for requirements on the boundary function which essentially cannot be lowered in Hölder classes $C^{k, \lambda}$.

\section{Numerical results}

We solved the following two problems using the finite difference method to justify the obtained theoretical results.

Let $\Pi=\{(x, y) \mid 0<x<1,-0.25<y<0.25\}$, and let $\gamma$ be the boundary of $\Pi$.

PROBLEM 6.1.

$$
\Delta u=0 \quad \text { on } \Pi, \quad u=v(r, \theta) \quad \text { on } \gamma
$$

where

$$
v(r, \theta)=r^{1+\lambda} \cos (1+\lambda) \theta, \quad \frac{1}{2}<\lambda<1,
$$

is the exact solution of Problem 6.1 and $v \in C^{1, \lambda}(\gamma) ; r=|z|, \theta=\arg (z)$, and $z=x+i y$.

PROBLEM 6.2.

$$
\Delta u=0 \quad \text { on } \Pi, \quad u=w(x, y) \quad \text { on } \gamma
$$

where

$$
w(x, y)=\left(x^{2}-y^{2}\right) \tan ^{-1} \frac{y}{x}+2 x y \ln \sqrt{x^{2}+y^{2}}
$$

is the exact solution of Problem 6.2 and $w \in C^{1,1}(\gamma)$.

In both problems we solved finite difference equations by Gauss-Seidel iterations for $h=2^{-n}, n=3,4,5,6,7,8$. We request that the maximum successive error be reduced by a factor of $10^{-17}$ as a stopped criterion. To check the order of error in 
TABLE 1. $\Re_{n}$ when $\lambda=0.55,2^{1+\lambda}=2.92817139$.

\begin{tabular}{rlll}
\hline$h^{-1}$ & Five-point & Nine-point & "Five and nine"-point \\
\hline 16 & 2.768108 & 2.748222 & 2.959148 \\
32 & 2.878428 & 2.879657 & 2.912227 \\
64 & 2.911644 & 2.915787 & 2.923505 \\
128 & 2.922537 & 2.925058 & 2.927079 \\
256 & 2.926212 & 2.927392 & 2.927903 \\
\hline
\end{tabular}

TABLE 2. $\Re_{n}$ when $\lambda=2 / 3,2^{1+\lambda}=3.1748021024$.

\begin{tabular}{rlll}
\hline$h^{-1}$ & Five-point & Nine-point & "Five and nine"-point \\
\hline 16 & 2.962831 & 2.984583 & 3.227898 \\
32 & 3.101655 & 3.123321 & 3.161703 \\
64 & 3.147801 & 3.161648 & 3.170360 \\
128 & 3.164576 & 3.171495 & 3.173774 \\
256 & 3.170864 & 3.173974 & 3.174555 \\
\hline
\end{tabular}

TABLE 3. $\Re_{n}$ when $\lambda=0.75,2^{1+\lambda}=3.36358566$.

\begin{tabular}{rlll}
\hline$h^{-1}$ & Five-point & Nine-point & "Five and nine"-point \\
\hline 16 & 3.103846 & 3.165907 & 3.431869 \\
32 & 3.267131 & 3.309923 & 3.352983 \\
64 & 3.325364 & 3.349864 & 3.359334 \\
128 & 3.348067 & 3.360135 & 3.362606 \\
256 & 3.357195 & 3.362721 & 3.363349 \\
\hline
\end{tabular}

maximum norm for the finite difference method we use the quotient of maximum errors

$$
\Re_{n}=\frac{\max _{\Pi^{h}}\left|u_{2^{-n}}-u\right|}{\max _{\Pi^{h}}\left|u_{2^{-(n+1)}}-u\right|} .
$$

The results given in Tables $1-4$ for the Problem 6.1 show that $\Re_{n}<2^{1+\lambda}$ for increasing $n$ for all five-point, nine-point, and "five and nine"-point schemes. This property is also illustrated in Figures 1 and 2. These results are the numerical justification of Theorem 4.2 and Remark 5.2, that is, if the boundary function is from $C^{1, \lambda}(\gamma), 1 / 2<\lambda<1$, then the maximum error is bounded below by the quantity $h^{1+\lambda}$ on some sequence of values $h$ tending to zero. Therefore, the maximum error will converge to zero slower than $h^{2}|\ln h|$.

Table 5, Figures 3 and 4 are the results for Problem 6.2. Figure 4 is an enlargement of the piece $(n \geq 6)$ of Figure 3 by scaling $\Re_{n}$ with the formula $\left(\Re_{n}-3.99\right) \times 10^{5}$. These results are the numerical justification of Theorem 5.1. Indeed, for the "five and nine"-point scheme $\Re_{n} \geq 4$ when $n \geq 4$ which corresponds to an error of estimation of the order of $O\left(h^{2}\right)$, and for the five-point and nine-point schemes $4>\Re_{n} \geq$ $4 n /(n+1)$ which corresponds to an error of estimation of $O\left(h^{2}|\ln h|+1\right)$. 

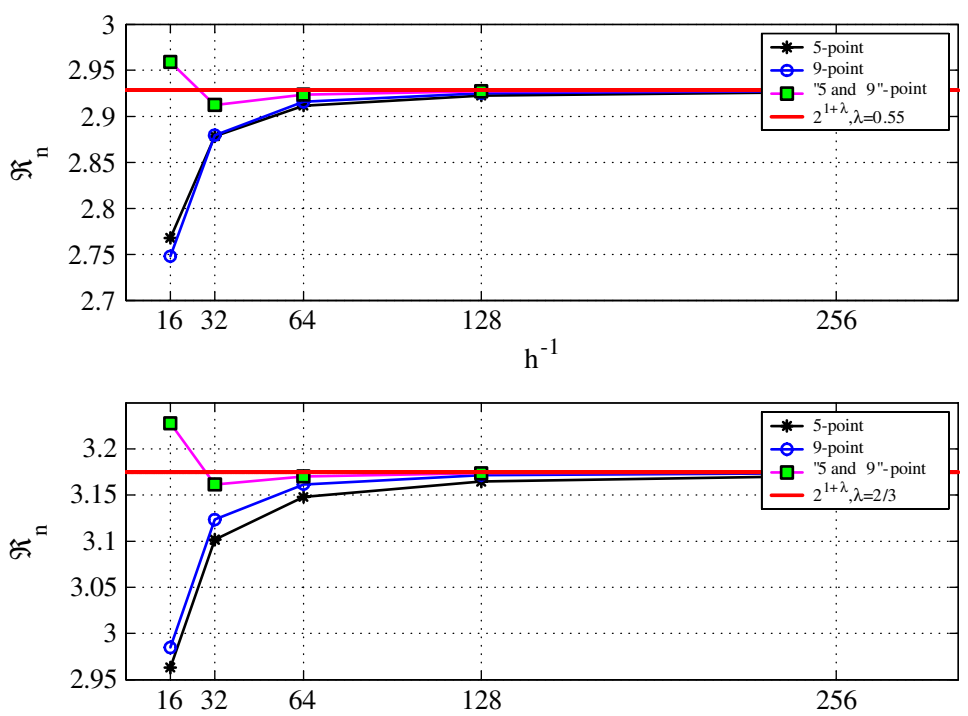

FiguRE 1. $\Re_{n}$ for Problem $6.1, \lambda=0.55$ (top), $2 / 3$ (bottom).
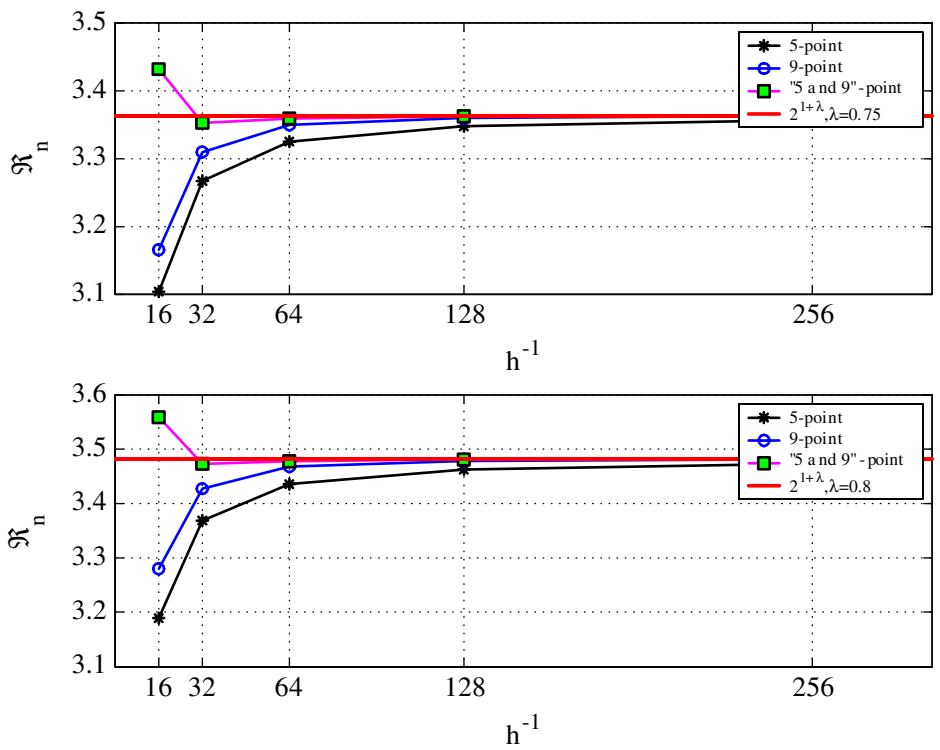

FIGURE 2. $\Re_{n}$ for Problem 6.1, $\lambda=0.75$ (top), 0.8 (bottom). 


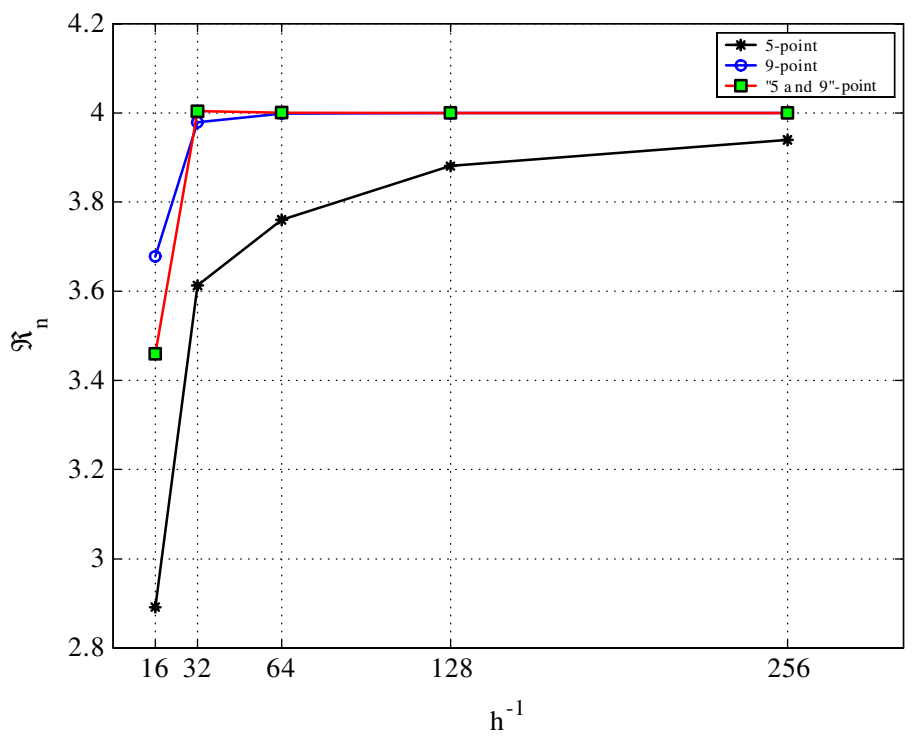

FIGURE 3. $\Re_{n}$ for Problem 6.2.

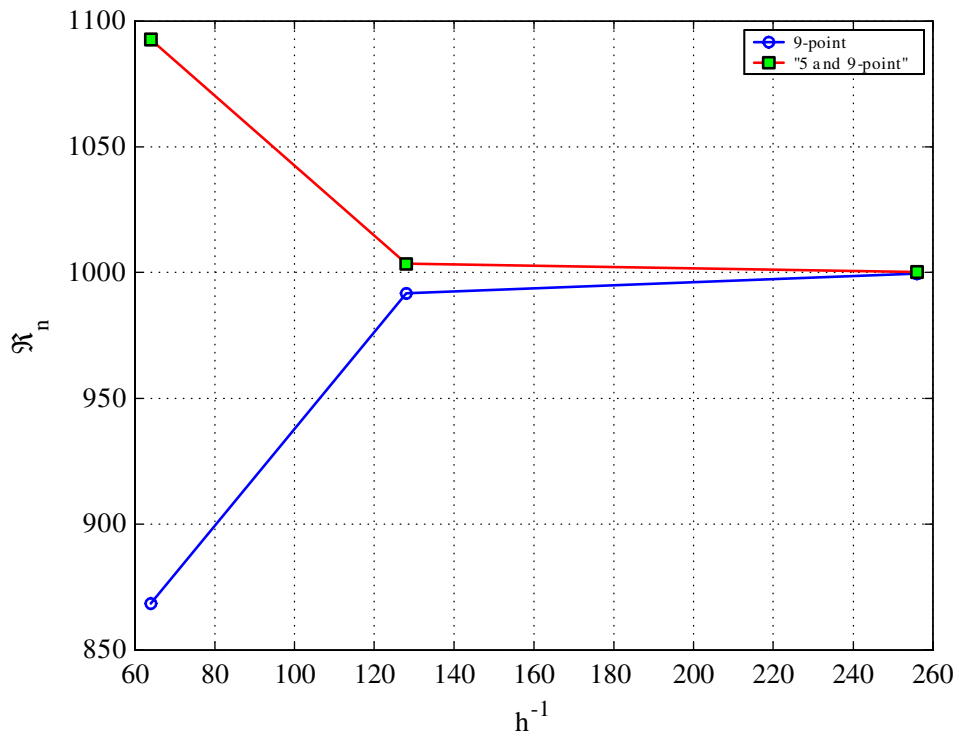

FIGURE 4. $\Re_{n}$ for Problem 6.2. 
TABLE 4. $\Re_{n}$ when $\lambda=0.8,2^{1+\lambda}=3.48220225$.

\begin{tabular}{rlll}
\hline$h^{-1}$ & Five-point & Nine-point & "Five and nine"-point \\
\hline 16 & 3.188975 & 3.280010 & 3.559057 \\
32 & 3.368577 & 3.427208 & 3.473288 \\
64 & 3.435312 & 3.468138 & 3.478078 \\
128 & 3.462390 & 3.478664 & 3.481253 \\
256 & 3.473726 & 3.481316 & 3.481974 \\
\hline
\end{tabular}

TABLE 5. $\Re_{n}$ for Problem 6.2.

\begin{tabular}{llll}
\hline$h^{-1}$ Five-point & Nine-point & "Five and nine"-point & \\
\hline 16 & 2.89131843 & 3.67836720 & 3.46006310 \\
32 & 3.61269139 & 3.97914610 & 4.00397185 \\
64 & 3.76022496 & 3.99868404 & 4.00092659 \\
128 & 3.88094591 & 3.99991756 & 4.00003447 \\
256 & 3.93998220 & 3.99999483 & 4.00000125 \\
\hline
\end{tabular}

\section{Conclusions}

A theoretical error analysis of the finite difference schemes in solving Laplace's equation on rectangular domain is given. It is shown that the uniform estimate of the error of the nine-point solution is of the order of $O\left(h^{2}(|\ln h|+1)\right.$, a similar order as the five-point solution, when the given boundary function is from $C^{1,1}$. To improve the accuracy, the nine-point scheme should be replaced in the grids nearest to the boundary of the rectangle by the five-point scheme ("five and nine"-point scheme). It is also proved that for the uniform error obtained for the nine-point and "five and nine"-point schemes, the requirements imposed on the boundary functions cannot be lowered in $C^{k, \lambda}$. Numerical experiments are illustrated to support the analysis made.

The obtained results can be used to justify the finite difference method for some version of domain decomposition methods, for composite grid methods, and for the combined methods to approximate a nonsmooth solution of Laplace's equation.

\section{References}

[1] J. H. Bramble, B. E. Hubbard and V. Thomee, "Convergence estimates for essentially positive type Dirichlet problems", Math. Comp. 23 (1969) 695-709.

[2] A. A. Dosiyev, "A block-grid method of increased accuracy for solving Dirichlet's problem for Laplace's equation on polygons", Comput. Math. Math. Phys. 34 (1994) 591-604.

[3] A. A. Dosiyev, "A fourth order accurate composite grids method for solving Laplace's boundary value problems with singularities", Comput. Math. Math. Phys. 42 (2002) 832-849.

[4] A. A. Dosiyev, "On the maximum error in the solution of Laplace equation by finite difference method", Int. J. Pure Appl. Math. 7 (2003) 229-241.

[5] A. A. Dosiyev, "The high accurate block-grid method for solving Laplace's boundary value problem with singularities", SIAM J. Numer. Anal. 42 (2004) 153-178.

[6] A. A. Dosiyev and S. Cival, "A combined method for solving Laplace's boundary value problem with singularities”, Int. J. Pure Appl. Math. 21 (2005) 353-367.

[7] L. V. Kantorovich and V. I. Krylov, Approximate methods of higher analysis (Noordhoff, Leiden, 1958). 
[8] Z. C. Li, Combined methods for elliptic problems with singularities, interfaces and infinities (Kluwer, Dordrecht, 1998).

[9] A. A. Samarskii, The theory of difference schemes (Marcel Dekker, New York, 2001).

[10] B. F. Smith, P. E. Bjorstad and W. D. Gropp, Domain decomposition: parallel multilevel methods for elliptic partial differential equations (Cambridge University Press, Cambridge, 1996).

[11] E. A. Volkov, "Differentiability properties of solutions of boundary value problems for the Laplace and Poisson equations on a rectangle", Proc. Steklov Inst. Math. 77 (1965) 101-126.

[12] E. A. Volkov, "On differential properties of solutions of the Laplace and Poisson equations on a parallelepiped and efficient error estimates of the method of nets", Proc. Steklov Inst. Math. 105 (1969) 54-78.

[13] E. A. Volkov, "The differential properties of the solutions of Laplace's equation, and the errors in the method of nets with boundary values in $C_{2}$ and $C_{1,1}$ ", Comput. Math. Math. Phys. 9 (1969) 97-112.

[14] E. A. Volkov, "On the method of composite meshes for Laplace's equation on polygons", Tr. Mat. Inst. Steklova 140 (1976) 68-102. 